\section{PTU-008 A REPRODUCIBLE, CLINICALLY-RELEVANT, INTENSIVELY- MANAGED, PIG MODEL OF ACUTE LIVER FAILURE FOR TESTING OF THERAPIES AIMED TO PROLONG SURVIVAL}

\section{doi:10.1136/gutjpl-2012-302514c.8}

${ }^{1} \mathrm{~K}$ Lee, ${ }^{*}{ }^{1} \mathrm{C}$ Palacios Jimenez, ${ }^{1} \mathrm{H}$ Alibhai, ${ }^{1} \mathrm{Y}-\mathrm{M}$ Chang, ${ }^{2} \mathrm{P}$ Leckie, ${ }^{1} \mathrm{~S}$ Priestnall, ${ }^{2} \mathrm{R}$ Mookerjee, ${ }^{2} \mathrm{R}$ Jalan, ${ }^{2} \mathrm{~N}$ Davies. ${ }^{1}$ The Royal Veterinary College, University of London, Hatfield, London, UK; ${ }^{2}$ UCL Hepatology, University College London Medical School, London, UK

Introduction Hospitalisations from acetaminophen poisoning are increasing (1999, $n=39045 ; 2010, n=52707$. UK, NHS admissions). For the most serious cases there are no effective therapies to assist recovery or prolong survival apart from liver transplantation, which remains a limited resource. We report a clinically-relevant, intensively-managed, model of ALF, which mimics the human condition and has a predictable survival time, for testing of new potential therapies.

Methods Nine, $30-40 \mathrm{~kg}$, female pigs were anaesthetised and instrumented for continuous monitoring and management of respiratory and cardiovascular systems and acid-base and electrolyte status, using standardised intensive care protocols and intermittent positive pressure ventilation. Intracranial pressure (ICP) was monitored but not treated. Six animals were induced to ALF with acetaminophen administered via an oroduodenal tube: a loading dose of $0.25 \mathrm{~g} / \mathrm{kg}$ was followed by hourly doses of $0.5-4.0 \mathrm{~g}$ adjusted according to serum acetaminophen concentrations. At irreversible ALF (defined as prothrombin time $>3$ times normal), continuous renal replacement therapy (CRRT) was initiated. Three animals acted as controls with initiation of CRRT at $20 \mathrm{~h}$ and termination at $40 \mathrm{~h}$.

Results Following onset of acetaminophen dosing, peak serum acetaminophen concentrations of $367 \pm 30 \mathrm{mg} / \mathrm{l}$ were achieved at $12 \mathrm{~h}$ and irreversible ALF at $19.3 \pm 1.8 \mathrm{~h}$. Death occurred predictably $12.6 \pm 2.7 \mathrm{~h}$ after irreversible ALF. Development of ALF was associated with progressive hypotension $(p<0.001)$ and metabolic acidosis $(p=0.001)$, not observed in controls. Mean arterial pressure (MAP) was maintained with aggressive fluid therapy, noradrenaline and terlipressin. Metabolic acidosis was corrected successfully with bicarbonate and CRRT. In ALF, there was significant $(p<0.001)$ rise in ICP compared to controls with sudden marked increase prior to death: at study end, ICP in ALF and controls was 41.2 $\pm 8.6 \mathrm{~mm} \mathrm{Hg}$ and $22.7 \pm 2.5 \mathrm{~mm} \mathrm{Hg}$ respectively. Death was preceded by abrupt increase in central venous pressure, fall in MAP and bradycardia. Histopathology confirmed moderate to marked acute centrilobular to midzonal hepatocyte degeneration and necrosis in ALF.

Conclusion A predictable model of ALF, with death due to multiorgan failure, has been successfully validated for translational studies for therapies designed to prolong survival in man.

Competing interests None declared.

\section{PTU-009 ALCOHOL-INDUCED LIVER TOXICITY IS ASSOCIATED WITH NEUTROPHIL DYSFUNCTION IN A NOVEL IN-VITRO MODEL OF ACUTE LIVER INJURY}

doi:10.1136/gutjnl-2012-302514c.9

\footnotetext{
${ }^{1,2} \mathrm{~L} J$ Markwick, ${ }^{1} \mathrm{E}$ Palma, ${ }^{1} \mathrm{~A}$ Riva, ${ }^{1} \mathrm{R}$ Williams, ${ }^{3} \mathrm{D}$ Clemens, ${ }^{2} \mathrm{D}$ Shawcross, ${ }^{1} S$ Chokshi. 'Institute of Hepatology, Foundation for Liver Research, London, UK; ${ }^{2}$ Institute of Liver Studies, King's College London at King's College Hospital, London, UK; ${ }^{3}$ University of Nebraska Medical Center and Veteran Affairs Medical Center, Omaha Nebraska, USA
}

Introduction Sepsis is a major cause of mortality in patients with alcohol-induced acute and chronic liver failure (ALF/CLF). Neutrophils are a major innate immune cell subset involved in the first line of defence against infection and circulating neutrophil dysfunction has been reported in patients with ALF/CLF. However, there is a paucity of understanding regarding the mechanisms involved in this dysfunction. In this study we aimed to characterise the precise relationship between neutrophil dysfunction and alcohol-induced liver damage with a novel in vitro model mimicking the in vivo interactions of neutrophils and hepatocytes.

Methods We cultured a well-characterised neutrophil-line HL-60 either directly with ethanol or with supernatants taken from ethanol metabolising-human hepatoma cell lines VL-17A (positive for alcohol-dehydrogenase and CYP2E1) cultured in the presence of safe levels $(10 \mathrm{mM})$ and toxic levels $(250 \mathrm{mM})$ of ethanol reflecting real-life human alcohol consumption for $24 \mathrm{~h}$. Neutrophil function was evaluated by TLR expression, chemotaxis, phagocytosis and respiratory burst assays. Cell supernatants were also collected for cytokine profiling and to quantitate levels of ammonia and ethanol metabolites. The effect of ethanol on the functional activities of neutrophils isolated from both normal and ALF/CLF patients will also be assessed.

Results Supernatants collected from the hepatoma line VL17A cultured with $250 \mathrm{mM}$ ethanol (representative of an alcohol binge) significantly reduced the phagocytic capacity of the HL-60-neutrophil line $(p<0.05)$. This was greater than the effect of the same concentration of ethanol applied directly to the neutrophils $(p<0.05)$. Our preliminary data also suggests that the metabolised ethanol inhibits chemotaxis of the HL60-neutrophil cells towards a gradient of fMLP. Furthermore, we observed a reduction of TLR4 expression.

Conclusion We describe a novel model for investigating the correlates of dysfunctional innate immunity during acute alcohol-induced liver injury. We identify that alcohol does impair neutrophil function directly but this is profoundly increased after hepatocyte alcohol metabolism implicating a causal link between liver injury and impairment of antibacterial neutrophil functions.

Competing interests None declared.

\section{PTU-010 COMPUTATIONAL MODEL PREDICTS THAT PORTOSYSTEMIC SHUNTING IS KEY TO THE DEVELOPMENT OF HYPERAMMONAEMIA IN LIVER CIRRHOSIS}

doi:10.1136/gutjnl-2012-302514c.10

${ }^{1,2} \mathrm{~L}$ Noiret, ${ }^{*}{ }^{3} \mathrm{~S}$ Baigent, ${ }^{2} \mathrm{M}$ Jover Cobos, ${ }^{2} \mathrm{R}$ Jalan. ${ }^{1}$ CoMPLEX, UCL, London, UK; ${ }^{2}$ Medicine, UCL Institute of Hepatology, London, UK; ${ }^{3}$ Mathematics, UCL, London, UK

Introduction Hyperammonaemia occurs in patients with advanced cirrhosis, which is also associated with a major redistribution of blood flow (hyperdynamic circulation). Previous investigations have sought to explain hyperammonaemia on the basis of metabolic adaptation (enzymatic derangements). We hypothesised that hyperammonaemia is centrally linked to the haemodynamic disturbances caused by portal hypertension. To test this hypothesis, we developed a theoretical model, which predicted arterial ammonia levels when organ blood flow is modulated.

Methods Assumptions on individual organ fluxes of ammonia (across the gut, liver, muscle, brain and kidney) were based on published arterio-venous differences and tracer kinetic data. In order to study the role of organ blood flow in isolation, we assumed that hepatic detoxification function and ammonia production were normal. A wide range of conditions was investigated (increased cardiac output, splanchnic vasodilation, low to high porto-systemic shunt fraction). In addition, we used scenarios of organ blood flow corresponding to Child Pugh A, B and C. Finally, we considered the effect of lowering ammonia release in the renal vein.

Results Hyperammonaemia developed when the fraction of gastrointestinal blood shunted was more than $65 \%$. The influence of 